\title{
Response to the Chief Medical Officer's Report on Specialist Training
}

\section{Hospital Doctors: training for the future}

\author{
Fiona Caldicott, President, The Royal College of Psychiatrists
}

This report was published at the end of April 1993 by a Working Group established in 1992. The Group was asked by the Secretary of State for Health to consider the current system of specialist medical training in the light of the EC medical directives. These determine that "specialist training" begins when full registration is obtained and continues until a Certificate of Completed Specialist training is awarded.

The Group's recommendations have the twin aims of meeting the requirements of European Community law, and ensuring that standards of specialist training are maintained.

The main implications of the proposals relate to a clear definition of specialist training, with defined starting and finishing points, and structured curricula. The arrangements for the first phase of training must provide sufficient flexibility to enable a trainee doctor to make an initial commitment to a broad range of specialties, and where he or she so chooses, to delay a final commitment to pursue a specialty training programme. There should also be competition and assessment on merit. Programmes will be determined by the Medical Royal Colleges, and the Regional Postgraduate Deans will be responsible for ensuring that effective NHS training is provided consistent with the new curricula. The programme will broadly subsume the present accreditation system, where one is in place, and align specialist training in the UK with practice in Europe.

It is stated that the continued development of more structured and intensive training programmes will make it possible to complete training sooner, thereby reducing the length of specialist training, while ensuring that standards are maintained. This will enable the duration of training to be reduced to seven years or less in most specialties.

The report also recommends that the registrar and senior registrar grades should be combined, and that consideration should be given to the introduction of a single training grade. This would help to avoid the lengthening of training which can occur because of delays in progressing from one grade to another.

The award of a Certificate of Completion of Specialist Training is recommended, thereby introducing a clearly defined end point to specialist training, which the Group has determined begins immediately after full registration, as required by European law. Only that experience and training which fulfils the requirements and meets the standards of the accrediting authority, compatible with independent practice, would be recognised for the award of a UK CCST.

The Group decided that the addition of the "CT" indicator to the Medical Register should be on a voluntary basis only. Information about an individual's specialist qualifications would then be available to the general public through the Medical Register.

The Group discussed the possibility of making the CCST, or its equivalent, from other EC member states, a requirement for specialist practice. It decided against this on the grounds that it might lead to doctors being restricted to procedures only within their own specialties. They also did not want to limit the flexibility of Advisory Appointment Committees, but recommended that guidance for these committees should be reviewed.

The Group was strongly opposed to the creation of a new career grade junior to consultant, but favoured a rapid increase in the number of consultants. This would lead to improved services for patients, and help to meet the need to replace the service contribution lost from doctors in training, as training programmes shorten and intensify. It would also help to avoid the problem of doctors who have been awarded the CCST being caught in the "gap" between completion of training and appointment to a consultant post.

The main body of the Group's recommendations could be implemented within a period of two to three years of being accepted by Ministers, and the expansion in the consultant grade might take place over a period of five years or so.

The Group has recommended the establishment of discussions between the Medical Royal Colleges, Postgraduate Deans and NHS management to facilitate the introduction of the changes. The Medical Royal Colleges must also consider the implications for research and academic medicine. 
Finally, it is recognised that the recommendations, if accepted, will have wide ranging implications for the way in which qualified medical staff will work in the future. However, the Department will be looking for a method to implement the recommendations which does not increase paybills beyond existing plans. Importantly, it is proposed that there should be greater mobility within the consultant grade.

\section{The implications for psychiatry Training programmes}

The progress that Colleges and their Faculties are making in developing more organised training programmes is noted in the report. The opportunities thereby created for a significant reduction in the duration of training, without compromising standards, is welcomed. This should continue, and should lead to most doctors being able to obtain their CCST within seven years.

It can be stated with confidence that training programmes in psychiatry are already "organised". The length of training recommended by the Royal College of Psychiatrists is six to seven years, although the "bottleneck", which exists between the registrar and senior registrar grades, currently lengthens training inappropriately.

The Working Group suggests that curricular requirements for planned specialist training programmes should be specified by July 1994.

An Educational Strategy Working Group has been established, under the chairmanship of Dr Michael Harris, Sub-Dean, which will, through consulting all appropriate Committees and Sections in the College, revise our existing training programmes and their relationship to the Membership examinations.

The Working Group recommends that these programmes should be implemented by the end of 1995 .

This is entirely feasible in psychiatry as we are unlikely to make radical changes. The exhortation to monitor these changes carefully to ensure that standards are maintained is consistent with our existing policies. Indeed, it is through our training programmes that we have raised standards of psychiatric care and I am confident that this process will continue. Academic and research medicine should be considered with the Association of University Teachers of Psychiatry.

\section{General professional/basic specialist training}

The Working Group recommends that further consideration should be given to this period, by a working party convened by the GMC.

This should be welcomed by the College, if it will lead to greater flexibility in the recognition of experience in other parts of medicine undertaken by the would-be specialist. Training in and for general practice should also be facilitated.

\section{Certificate of completed specialist training}

This will be awarded by the GMC on advice, from the relevant Medical Royal College, that the doctor has satisfactorily completed specialist training, based on assessment of competence, to a standard compatible with independent practice and eligibility for consideration of appointment to a consultant post. It should not be awarded at an intermediate point. It is acknowledged that this must be distinguished from continuing medical education, which should extend throughout a doctor's career.

The College [through the Court of Electors] will ask the Joint Committee on Higher Psychiatric Training for advice about the criteria to be used for satisfactory completion of psychiatric training, and how competence is to be assessed formally. There is no support for an "exit" examination in psychiatry at present and no cogent argument has emerged for its further consideration. The current system for reviewing the progress of higher psychiatric trainees may require re-examination.

\section{The training grade structure}

The Working Group recommends that the Health Departments, following appropriate consultation, should reconsider the training grade structure with the aim of introducing a combined higher training grade to replace the registrar and senior registrar grades as soon as is practicable and in any case no later than the end of 1995. It should be considered if integration should proceed further once the GMC has determined the future place of general professional/basic specialist training within the overall specialist training framework.

This is the recommendation which could lead to the greatest upheaval in psychiatry if it is not challenged. It must be explained that registrars in psychiatry are not equivalent to registrars in many specialties. They are undertaking basic specialist training and it is only when they have obtained the MRCPsych that they obtain a senior registrar post and commence higher psychiatric, or specialist training, in one of the six recognised psychiatric specialties. It would, however, be practicable to merge the senior house officer and registrar posts in psychiatry, undertaking as they do general professional/basic specialist training. It may be that in the process of reviewing general professional training, pre$M R C P s y c h$ trainees can be subsumed in a renamed senior house officer grade.

\section{Expansion of the consultant grade}

It would not be possible, at the moment, to increase the number of consultants in psychiatry by converting registrar and/or senior registrar posts into consultant posts, as all the existing training posts are needed for the foreseeable number of consultant vacancies.

Mobility within the consultant grade should be welcomed as this should lead to a healthier workforce, 
as long as it does not suggest that greater use of shortterm contracts is desirable.

\section{Conclusion}

There is much to be welcomed in this report, seeking to ensure as it does that "training" means precisely that, while upholding the standards of practice of those deemed eligible for a consultant post. In psychiatry we must protect our achievements thus far and demonstrate that we provide most of what is required already, and are prepared to comply where we do not.
It is a pity that we are misrepresented on page 33 of 'Training for Specialist Practice'. There is no intention to reduce training in psychiatry to five to six years here. This comment is thought to be based on a misinterpretation of discussion about a possible target time. In most countries in Europe, there is a wish to lengthen the existing duration of training, and EC countries each determine the period of training in psychiatry required for their specialist certificate.

See also pages $610-612$

Psychiatric Bulletin (1993), 17, 579-581

\title{
Keynotes
}

\section{Tomlinson on alcohol and drugs}

\author{
Hamid Ghodse, Professor of Psychiatry, St George's Hospital Medical School, \\ Tooting, London SW17 0RE
}

\section{Recognition of the problem}

Sir Bernard Tomlinson's Report of the Enquiry into London's Health Service, Medical Education and Research (1992) starts with an excellent analysis of the particular problems associated with providing health care in the capital with its high density and turnover of population and disproportionate burden of severe deprivation. He emphasises the particular problems that arise because of the flow into London of vulnerable people with mental illness, drug addiction problems and alcoholism, many of whom are homeless. The consequent difficulties are compounded by the fact that primary health care and community services are poorly developed in comparison with elsewhere in the country and may not be easily accessible to those who need them most. Inadequacies in comprehensive service provision contribute to the high usage of accident and emergency departments as providers of primary health care, where community nursing services are expensive because of higher staff costs and where the characteristics of the patient population referred to above, make maintaining effective contact very difficult.

All of this is familiar to those who work in London. It is Tomlinson's proposals for addressing the problems, and the impact that these solutions might have on substance misusers, that are, therefore, of interest.

\section{Developing primary health care}

The first recommendation is for a gradual and systematic transfer of resources from the acute sector to community health service and family health service budgets. This carries the risk that in the absence of extra, bridging finance, some acute hospitals could close before community provision is properly developed.

\section{Hospital closures}

Secondly, hospital closures, such as those recommended in the Report, would mean that accident and emergency services would be concentrated on fewer sites. Although the Enquiry emphasised the need for good access to accident and emergency departments, this was assessed in terms of the time travelled by road transport to a particular hospital. This approach largely ignores the needs of drug and alcohol users who attend accident and emergency departments regularly and who may not have access to transport. Substance misusers may not be registered with a general practitioner and any reduction in the number of accident and emergency departments will reduce the availability of an essential primary health care service, on which this group place great reliance. 